\title{
A bioinformatic evaluation of potential allergenicity of 85 candidate genes in transgenic organisms
}

\author{
ZOU ZeHong ${ }^{1 \dagger}$, HE Ying $^{1 \dagger}$, RUAN Lin $^{1 \dagger}$, SUN BaoQing ${ }^{1,2}$, CHEN HuiFang $^{1}$, CHEN De $^{1}$, \\ LIU ShiMing ${ }^{1}$, YANG XiaoGuang ${ }^{3} \&$ TAO AiLin ${ }^{1 *}$ \\ ${ }^{1}$ Guangzhou Municipal Key Laboratory of Allergy \& Clinical Immunology, Allergy Research Branch of the State Key Laboratory of Respiratory \\ Disease, the Second Affiliated Hospital of Guangzhou Medical University, Guangzhou 510260, China; \\ ${ }^{2}$ Guangzhou Institute of Respiratory Diseases, the First Affiliated Hospital of Guangzhou Medical University, Guangzhou 510120, China; \\ ${ }^{3}$ National Institute for Nutrition and Food Safety, Chinese Centre for Disease Control and Prevention, Beijing 100050, China
}

Received June 10, 2011; accepted December 19, 2011

\begin{abstract}
The potential of genetically modified organisms (GMOs) to increase allergenicity or predispositions to allergies has attracted much attention. An approach that will properly and holistically evaluate the allergenicity of GMOs is yet to be found. Here, 85 transgenes that have been reported in both international and domestic studies during recent years are summarized case by case; 49 of the transformed genes were from plant sources and 36 were from animal sources. EVALLER ${ }^{\mathrm{TM}}$, a web server for the in silico assessment of potential protein allergenicity, was used to evaluate the potential of the transgenic proteins as allergens. The biomedical journals listed in Highwire (http://highwire.stanford.edu/) were searched and reviewed to decipher whether any of the transformed genes were linked to allergenicity or human health. The EVALLER analysis identified 5 allergenic genes, whilst our literature review found 11 genes that were either related to allergic cases or to clinical adverse events; all 16 of these genes have been used in GMOs. The analysis pathway that we have developed can help guide the selection of genes to be used in genetic modification. The pathway also provides a paradigm for allergenicity analysis of transgene candidates.
\end{abstract}

genetically modified organism, allergenicity, transgene, software, literature review

Citation: Zou Z H, He Y, Ruan L, et al. A bioinformatic evaluation of potential allergenicity of 85 candidate genes in transgenic organisms. Chin Sci Bull, 2012, 57: 1824-1832, doi: 10.1007/s11434-012-5149-4

A genetically modified organism (GMO) is a novel organism whose genetic material has been altered using transgenic biotechnology. Examples of GMO techniques include, but are not limited to, transferring of foreign genes from viruses, bacteria or non-food species, and transferring favorable or resistance genes from one food species to another food species or to one with less favorable target properties. GMOs have been shown to have incomparable advantages over traditionally bred products. GMOs break through the biological species concept, and consequently, more and more are being developed and put to widespread use. However, alongside the significant socioeconomic benefits that are

These authors contributed equally to this work

*Corresponding author (email: taoailin@gzhmc.edu.cn) generated, the biomedical safety of GMOs has increasingly come under public scrutiny. Safety evaluations prior to a GMO becoming commercial availability can be important in eliminating or minimizing the negative impacts and distrust of GMOs, and the risks of untoward food reactions and gastrointestinal disease could be reduced. The potential for increased allergenicity or predisposition to allergies caused by GMOs harboring novel proteins (as in the transgenic plants and animals that could become the mainstay of food sources for human), has attracted much attention from governments and communities worldwide. Therefore, an approach for the proper and overall evaluation of the allergenicity of each of the exotic gene introduced into GMOs remains an important topic to be addressed.

The aim of the present study was to summarize the aller- 
genicity of target genes that have been used in GMOs both internationally and domestically in recent years. We included a total of 85 transformed genes, 49 from plant sources and 36 from animal sources, in the study. These genes were retrieved from a bioinformatics databank, their protein coding regions were identified and the deduced amino acid sequences were evaluated for potential allergenicity. Literature on these 85 transformed genes was searched and reviewed to decipher whether or not they had been linked to allergenicity or human health. The information obtained could be used to guide the selection of genes to be used in genetic modification, as well to provide a paradigm for the allergenicity analysis of transgenes.

\section{Materials and methods}

\subsection{Data source of the transgenes}

International and domestic studies on GMOs published in recent years for which the full-text articles were available in the common literature databases (Highwire, PubMed and CNKI) were identified using the search terms "genetically modified organism", "transgenic organism" and a Chinese key word "transgenic gene". When there was redundant data on any individual gene (a gene with identical primer sequences and gene name) because, for example, it had been transformed to a variety of species, only one of the related articles was selected in our study and the remaining articles were excluded.

\subsection{Acquisition of information on the transgenes}

All bioinformatic data including primer sequences and gene names were extracted from the full-texts and searched against the nucleotide sequence database available at the National Center for Biotechnology Information (NCBI) using the Basic Local Alignment Search Tool (BLAST) to acquire the nucleotide sequences of the genes and their annotated pro- tein coding regions (CDSs). The CDS sequences were used to retrieve in-depth information from the Universal Protein Resource (UniProt) database; the Uniprot ID number and the corresponding amino acid sequence in FASTA format was extracted for each transgene.

\subsection{E-testing of protein allergenicity}

"E-testing of protein allergenicity" was performed using the software EVALLER ${ }^{\mathrm{TM}}$ (version 2.0) [1], a web server for the bioinformatics assessment of protein allergenicity. This tool is available on the "Food Safety" page (http://www.slv. se/en-gb/Group1/Food-Safety/e-Testing-of-protein-allergeni city/) of the National Food Agency, Sweden. Genes that were labeled as allergenic were further investigated in a number of common allergen databases (Table 1) to determine whether or not the allergens have been well-documented.

\subsection{Retrieval of medical reports with negative com- ments on transgenes}

Medical articles related to the transgenes were searched for in the biomedical journals listed in Highwire (http://www. highwire.org) using the gene names or their abbreviations, obtained as described in Section 1.2 above, as keywords and setting "include PubMed" as the filter mode. The full-texts of papers with abstracts containing negative comments on any of the transgenes were downloaded and saved for further confirmation.

\section{Results}

\subsection{Collection of the transgenes}

A search of transgene data (as of July 1, 2010) yielded 85 hits, 49 from plant sources and 36 from animal sources. The hosts for the transgenes included 13 plant species: rice, soybean, wheat, corn, rape (including Brassica napus), potato,

Table 1 Databases used in this study

\begin{tabular}{ll}
\hline \multicolumn{1}{c}{ Database } & \multicolumn{1}{c}{ Website } \\
\hline EVALLER & http://www.slv.se/en-gb/Group1/Food-Safety/e-Testing-of-protein-allergenicity/ \\
GenPept & http://www.ncbi.nlm.nih.gov/sites/entrez?db=protein \\
UniProt/SwissProt & http://www.uniprot.org \\
IUIS allergens & http://www.allergen.org \\
Allergen database & http://allergen.csl.gov.uk/ \\
Allergome & http://www.allergome.org/ \\
Allermatch & http://www.allermatch.org \\
SDAP-structural database of allergenic proteins & http://fermi.utmb.edu/SDAP/ \\
CSL allergen & http://www.fera.defra.gov.uk/ \\
FARRP & http://allergenonline.com \\
\hline
\end{tabular}


the tetraploid Robinia pseudoacacia, white clover, cotton, rye, tomato and tobacco; and 4 animal species: pig, cattle, sheep (including domestic sheep and goat) and mouse. The characters of the transgenes from plant sources mainly include the capacity for resistance to adversities (diseases, pests, drought and land salinization), aging, low temperature and insecticides, or their improvement to species quality, photosynthesis, bioavailability of limited elements from the soils, and their content of elements deficient in the human body. The transgenes from animal sources covered a range of benefits such as therapeutic effects for human diseases, and improved qualities and accelerated growth of the transgenic products. Detailed information on the sources, hosts, functions and use of all the transgenes collected is summarized in Table 2 and in Tables S1 and S2. The plant-based cell cycle regulatory protein cyclAt gene (ID \#1 transgene in Table S1) originating from Arabidopsis, for example, could be useful for modulation of growth and development in plants, and the animal-based HSA gene (ID \#8 transgene in Table 2) which was transformed from human to sheep, cattle and pig, could play a major role in the treatment of massive blood loss, burns, shock, edema and hypoalbuminemia, and may also be important in the manufacture of protective agents or stabilizers for various biological products and biochemical reagents.

\subsection{E-testing of allergenicity of the transgenes}

The transgene names and the sequences of the corresponding primers that were available in the full-texts were obtained.

Table 2 Summary of the transforming genes identified using our analysis pathway to be involved in allergenicity or adverse events ${ }^{\text {a) }}$

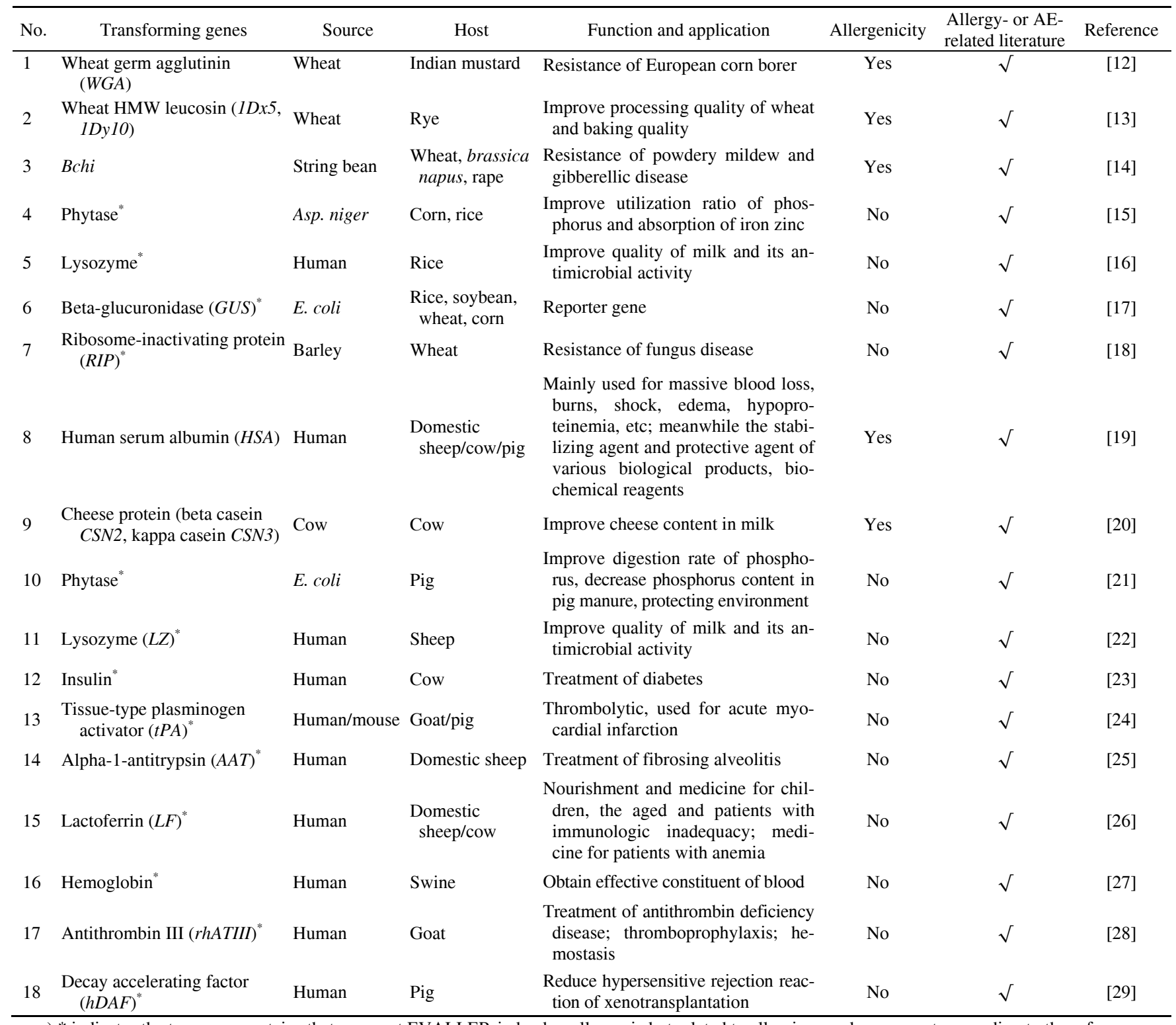

a) * indicates the transgene proteins that were not EVALLER-judged as allergenic but related to allergies or adverse events according to the references. 
For each gene, a consensus motif was generated by concatenating the forward primer to the reverse complemented sequence of the reverse primer with a string of N's between them. The consensus sequences were used to query the nucleotide sequence database at NCBI using the BLASTn program and all the sequences that matched a particular consensus motif were downloaded. Sequences with species origin and gene name consistent with what was described in the full-texts were selected as the target sequences. Using this procedure we obtained 85 full-length coding sequences of transgenes from which we generated the deduced protein sequences using a gene translation tool. The protein sequences were saved in FASTA format after confirming with NCBI/BLASTp program that the back-translations produced the original target sequences.

E-testing of allergenicity was performed using the deduced protein sequences of the transgenes. For example, the FASTA sequence of the HSA gene (ID \#8 transgene in Table 2), was pasted into the acceptor window of EVALLER 2.0 and the results of the allergenicity assessment were generated (Figures 1 and 2). The red-barred query shown in Figure 1 is an indication that the tested protein is an allergen. The five filtered length-adjusted allergen peptides (FLAPs) that best matched human serum albumin, are given in Figure 2. The results of the e-testing helped determine the allergenicity of some protein and allowed the error by which a misjudgment was made to be quantified. EVALLER also indicted that if the HSA gene was not allergenic then it would be non-allergenic in $0.0 \%$ of the times; this may be interpreted as a full confirmation on the allergenicity of HSA. Human serum albumin (HSA) has typically been used as a non-allergenic negative control or stabilizer in allergen assays and in other experiments [30]. The allergen prediction result for HSA was supported by the earlier observation that HSA may arouse allergy after being conjugated with plicatic acid or other haptens [31].

The results of the evaluation for allergenicity of the plant-based cell cycle regulatory protein (ID \#1 transgene in
Table S1) are shown in Figures 3 and 4. A difference to be noted is the green-barred query which indicated that the protein was predicted to be non-allergenic; however, the 5 best FLAPs shown in Figure 4 have an error possibility of only $6.8 \%$. An alternative interpretation is that the risk of committing an error could be greater than $5 \%$ when the cell cycle regulatory protein was considered to have no allergenicity. Further validation of these findings in future studies is warranted.

\subsection{Consideration of a special case}

Cowpea trypsin inhibitor (CPTI, ID \#5 transgene in Table $\mathrm{S} 1$ ), a gene with insect-resistant capacity that has been transformed into a number of crops, is generally believed to have no allergenicity. However, the sequence with the signal peptide intact was determined as definitely allergenic by EVALLER. It has been suggested that the CPTI signal peptide in cells of transgenic plants may deliver novel insect-resistant fusion proteins into the endoplasmic reticulum (ER) before it is recognized and truncated by some recognition mechanism [32]. Removal of the signal peptide was associated with the EVALLER prediction of the non-allergenicity of CPTI. If CPTI in transgenic products can be confirmed to exist only in the form of the mature peptide (containing no signal peptides), then this outcome of etesting with EVALLER may eventually shed light upon the allergenicity of the products of this transgene.

\subsection{Literature review reveals false negatives predicted by EVALLER}

Of the 49 plant-based transgenes only 3, wheat germ agglutinin (ID \#1 transgene in Table 2), wheat HMW leucosin (ID \#2 transgene in Table 2) and Leguminosae chitin synthetase (ID \#3 in Table 2) and, of the 36 animal-based transgenes only 2, human serum albumin (ID \#8 transgene in Table 2) and cheese protein (ID \#9 transgene in Table 2)

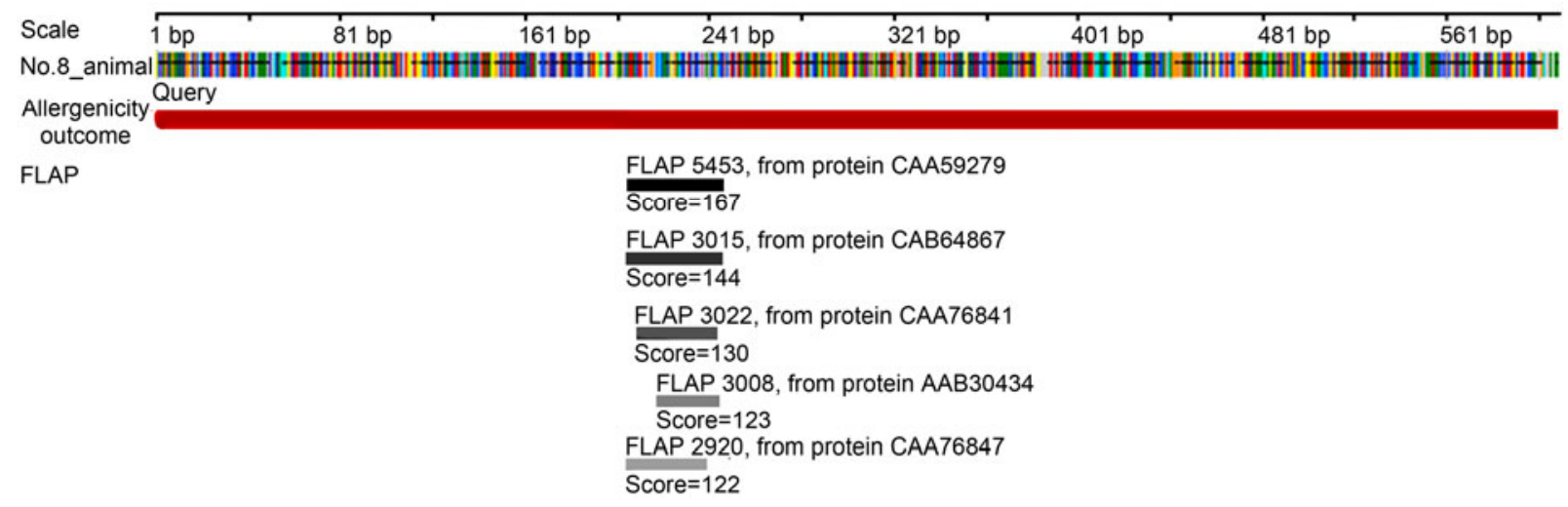

Figure 1 Evaluation of the allergenicity of the human serum albumin gene (ID \#8 transgene of animal source in Table 2) using EVALLER. The EVALLAR assignment of a red bar at the top of the output page represents a positive outcome ("allergen"); five filtered length-adjusted allergen peptides (FLAPs) were returned; the FLAP that best matches the target gene is the darkest. 


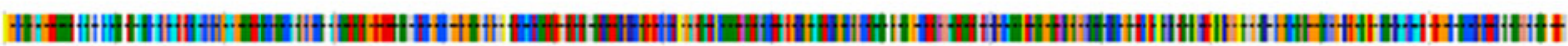

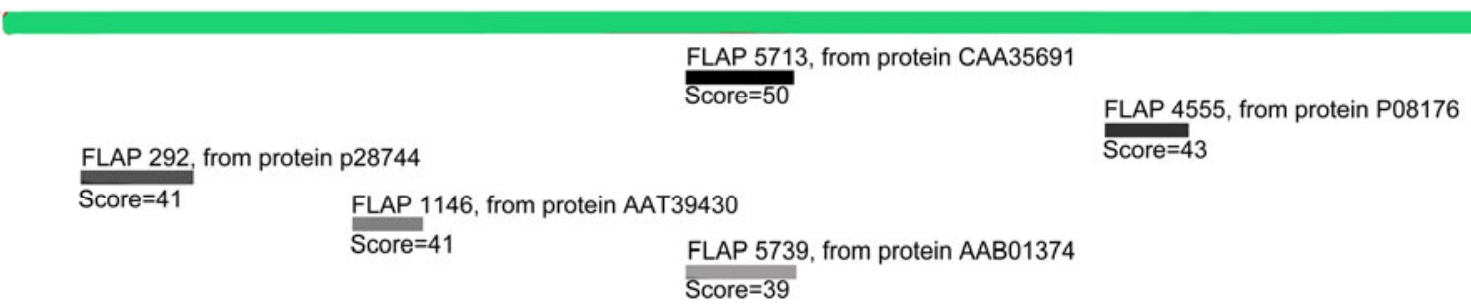

Figure 3 Evaluation of the allergenicity of the cell cycle regulatory protein gene (ID \#1 transgene of plant source) using EVALLER. The EVALLAR assignment of a green bar at the top of the output page represents a negative outcome ("non-allergen"); five filtered length-adjusted allergen peptides (FLAP) were returned; the FLAP that best matched the target gene is the darkest.

were predicted by EVALLER to be allergens; the remaining transgenes were predicted to be non-allergenic. However, our review of the literature revealed a number of allergies or adverse events that were associated with several of the transgene proteins that were not EVALLER-judged as allergenic. These proteins are identified with an asterisk $(*)$ in Table 2. The use of these 11 transgenes in GMOs should therefore be approached with caution.

For instance, although the antithrombin III protein (ID \#17 transgene in Table 2) was not predicted to be allergenic, in one study, it was recommended that it was not to be used in patients with severe disorders [33].

Thioredoxin, one of the proteins predicted with definite allergenicity [34], should be avoided as a candidate for gene transformation. However, transformation of its antisense gene could presumably be less allergenic and our review of the literature indicated that thioredoxin may reduce disulfide bonds and thereby lower the allergenicity of certain transgenic products [35]. This may be interpreted as the high allergenicity of a product being reversed by the antisense gene of thioredoxin. Therefore, the antisense transformation or knockout of thioredoxin needs special considerations when it is used in GMOs.

\section{Discussion}

In recent years, the safety profiles of genetically modified organisms (GMOs) have drawn much attention from governments, scientists and the public worldwide. Safety evaluation has been the only gateway for transgenic products to be marketed and put on dining-tables. Among all items listed in the safety evaluation, allergenicity of GMOs appears to be particularly important. A major example is the one of Pioneer Hi-Bred International Inc. which, in 1996, successfully transferred the $2 \mathrm{~S}$ albumin gene from Brazil nuts (Bertholletia excelsa) into soybeans to improve the nutritional quality of the latter. Unfortunately for them, the radioallergosorbent assay revealed this gene to be an allergen. Because the soybeans with $2 \mathrm{~S}$ albumin gene were highly likely to cause allergic reactions in humans, they were eventually not approved for commercial availability [36] becoming instead a great waste of the company's substantial investments in labor, material resources and finance. In 2001, the US Center of Disease Control and Prevention (CDC) filed around hundred cases of immune response allegedly associated with the consumption of a transgenic corn containing the insecticidal protein CRY9C. The issue of allergenicity to CRY9C was left unresolved because the link was not supported by the ELISA test carried out by the Food and Drug Administration [37]. These examples underline the value of using clinical evidences in the assessment of allergenicity.

In the present study, a total of 85 transgenes along with their sources, hosts, functions and uses were summarized, paying special attention to any reported allergenicity and related adverse effects. The algorithm that we developed to create the summary warrants standardization in future studies. First, medical literature and gene databases were searched to acquire bioinformatic data for the 85 transgenes. Evaluation of allergenicity of the deduced protein sequences was then performed using EVALLER 2.0. Five of the 85 transgenes, wheat germ agglutinin, wheat HMW leucosin, string bean chitinase, human serum albumin and cheese protein were immediately determined to be allergens. Further analysis showed that the allergenicity of cowpea trypsin inhibitor may vary depending upon whether or not the signal peptide was present. In addition, our study of the literature identified 11 transgenes ( $\beta$-glucuronidase, phytase, lysozyme, ribosomal passivate protein, antithrombin III, tissue plasminogen activator, $\alpha$-antitrypsin, lactoferrin, insulin, hemoglobin, and decay accelerating factor) that were related to allergies or clinically adverse events in a series of reports; all 11 transgenes had been determined to be non-allergenic by EVALLER. The various procedures in the analysis pathway apparently can complement each other, and could be used effectively at the beginning of any project to the transformation of a potentially harmful gene. This would avoid a great waste of early substantial investments and help reduce public distrust of GMOs.

The weight-of-evidence approach proposed by the Codex Alimentarius Commission guidelines (2003) has been 


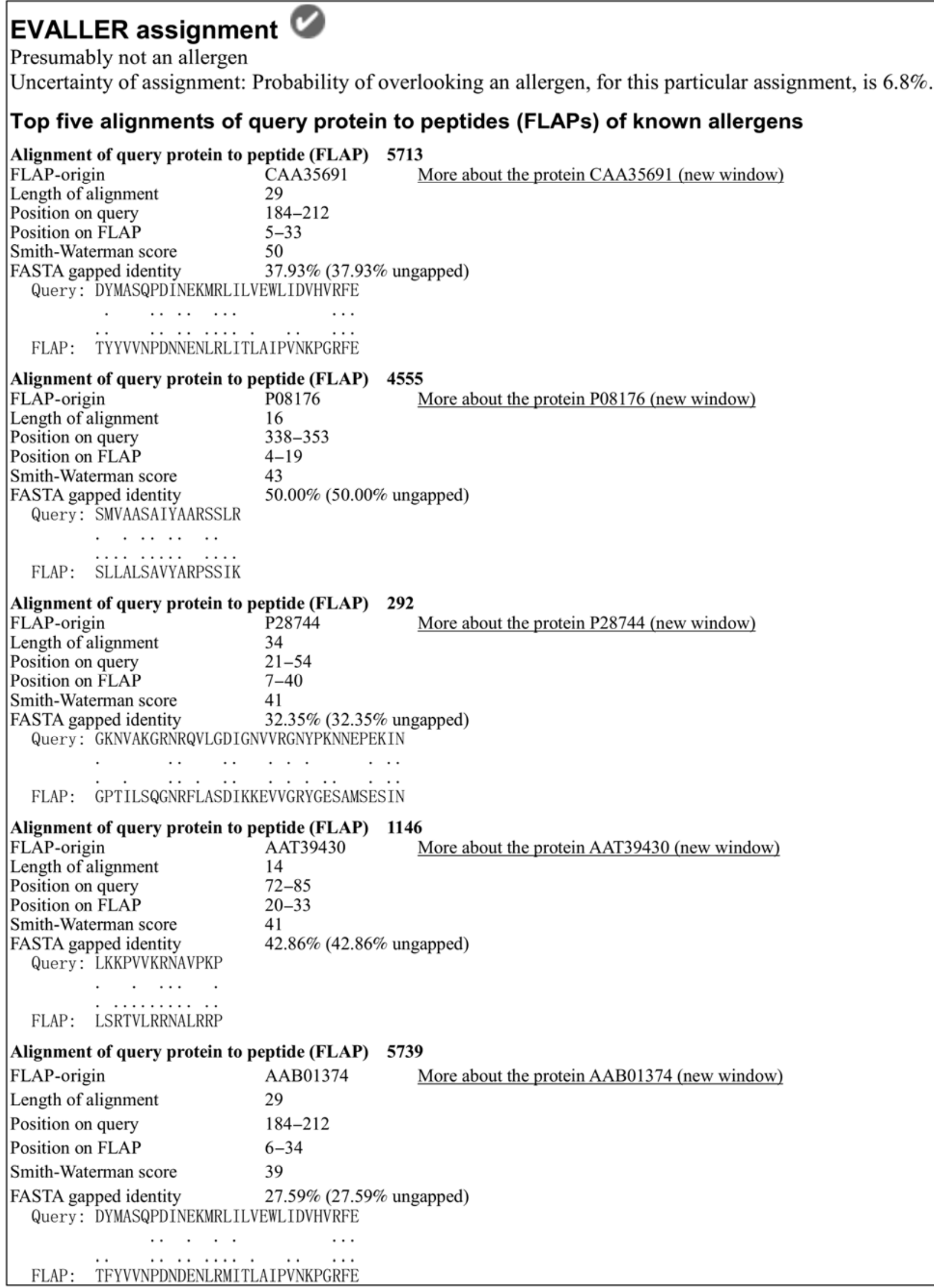

Figure 4 Evaluation of the allergenicity of cell cycle regulatory protein gene (cyclAt, ID \#1 transgene of plant source in Table S1) showing the 5 bestmatching FLAPs. 
partially accepted. The guidelines require that assessments holistically estimate the risk of allergenicity in transgenic products based on multiple lines of evidence, including the history of exposure and safety of the gene(s) source, protein structure, stability to pepsin digestion in vitro, an estimate of exposure of the novel protein(s) to the gastrointestinal tract where absorption occurs, specific IgE binding studies or skin prick testing, and when appropriate, animal models and targeted sera screening [38]. However, such approaches for predicting protein allergenicity have not been thoroughly evaluated or validated [38] nor have any one of them been confirmed in humans [39]. Herman et al. [40] showed an inconsistency between two criteria widely used for database queries in allergenicity assessment. In their study, allergen-sequence pairs that shared only an 8-amino-acid identity and not $>35 \%$ identity over 80 amino acids were unlikely to be cross-reactive allergens. In contrast, sequence pairs with $>35 \%$ identity over 80 amino acids were likely to be comparably allergenic.

EVALLER 2.0 includes 578 types of allergens and 2890 allergen-representative peptides [1]. This tool reliably assesses the allergenicity of transgene proteins using identities over 24 amino acids. On the one hand, sequence similarity may provide a preliminary judgment of allergenicity because sequential determinants play a major role in the allergenicity of a protein; however, on the other hand, conformational determinants are another important factor in allergy and these are not accounted for in the analysis of sequence similarity. Serological or other immunological tests should follow any bioinformatics study, to investigate whether the target protein could bind with a specific IgE and to obtain a more accurate evaluation for allergenicity of the GMOs consumed by humans. This is illustrated in our study in which we were able to identify the 11 allergenic transgenes not determined by EVALLER based on allergies or clinically adverse events reported in the literature.

This work was supported by the National Science and Technology Major Project of China (2008ZX08011-005, 2009ZX08011-004B), the National Natural Science Foundation of China (30771240) and the Medical Scientific Research Program of Guangdong Province (A2008284). We thank 20 members from Guangzhou Municipal Key Laboratory of Allergy and Clinical Immunology for their assistance on data collecting and allergenicity evaluation of the transgenes.

1 Barrio A M, Soeria-Atmadja D, Nistér A, et al. EVALLER: A web server for in silico assessment of potential protein allergenicity. Nucleic Acids Res, 2007, 35(Suppl 2): W694-W700

2 Miotto O, Tan T W, Brusic V. Rule-based knowledge aggregation for large-scale protein sequence analysis of influenza A viruses. BMC Bioinformatics, 2008, 9(Suppl 1): S7

3 Jain E, Bairoch A, Duvaud S, et al. Infrastructure for the life sciences: Design and implementation of the UniProt website. BMC Bioinformatics, 2009, 10: 136

4 McMillan L, Martin A. Automatically extracting functionally equivalent proteins from SwissProt. BMC Bioinformatics, 2008, 9: 418

5 Chapman M D, Pomes A, Breiteneder H, et al. Nomenclature and structural biology of allergens. J Allergy Clin Immunol, 2007, 119:
$414-420$

6 Bairoch A, Boeckmann B, Ferro S, et al. Swiss-Prot: Juggling between evolution and stability. Brief Bioinform, 2004, 5: 39-55

7 Lopata A L, O'Hehir R E, Lehrer S B. Shellfish allergy. Clin Exp Allergy, 2010, 40: 850-858

8 Fiers M W, Kleter G A, Nijland H, et al. Allermatch, a webtool for the prediction of potential allergenicity according to current $\mathrm{FAO} /$ WHO Codex alimentarius guidelines. BMC Bioinformatics, 2004, 5: 133

9 Ivanciuc $\mathrm{O}$, Midoro-Horiuti $\mathrm{T}$, Schein $\mathrm{C} \mathrm{H}$, et al. The property distance index PD predicts peptides that cross-react with $\operatorname{IgE}$ antibodies. Mol Immunol, 2009, 46: 873-883

10 Brusic V, Millot M, Petrovsky N, et al. Allergen databases. Allergy, 2003, 58: 1093-1100

11 Goodman R E, Hefle S L, Taylor S L, et al. Assessing genetically modified crops to minimize the risk of increased food allergy: A review. Int Arch Allergy Immunol, 2005, 137: 153-166

12 Kanrar S, Venkateswari J, Kirti P, et al. Transgenic indian mustard (Brassica juncea) with resistance to the mustard aphid (Lipaphis erysimi Kalt.). Plant Cell Rep, 2002, 20: 976-981

13 Altpeter F, Popelka J C, Wieser H. Stable expression of $1 D x 5$ and 1Dy10 high-molecular-weight glutenin subunit genes in transgenic rye drastically increases the polymeric glutelin fraction in rye flour. Plant Mol Biol, 2004, 54: 783-792

14 Huang Y S, Li H M. Arabidopsis CHLI2 can substitute for CHLI1. Plant Physiol, 2009, 150: 636-645

15 Veum T L, Ellersieck M R. Effect of low doses of Aspergillus niger phytase on growth performance, bone strength, and nutrient absorption and excretion by growing and finishing swine fed corn-soybean meal diets deficient in available phosphorus and calcium. J Anim Sci, 2008, 86: 858-870

16 Yang D, Guo F, Liu B, et al. Expression and localization of human lysozyme in the endosperm of transgenic rice. Planta, 2003, 216: 597-603

17 Wang W Q, Li Y, Zhang Y Y, et al. Comparative expression analysis of three genes from the Arabidopsis vacuolar $\mathrm{Na}^{+} / \mathrm{H}^{+}$antiporter (AtNHX) family in relation to abiotic stresses. Chin Sci Bull, 2007, 52: $1754-1763$

18 Oldach K H, Becker D, Lorz H. Heterologous expression of genes mediating enhanced fungal resistance in transgenic wheat. Mol Plant Microbe In, 2001, 14: 832-838

19 Huang Y, Huang Z, Yan J B, et al. High expression of human serum albumin in milk of transgenic mice directed by the goat $\beta$-casein gene promoter region. Chin Sci Bull, 2001, 46: 582-585

20 Brophy B, Smolenski G, Wheeler T, et al. Cloned transgenic cattle produce milk with higher levels of beta-casein and kappa-casein. Nat Biotechnol, 2003, 21: 157-162

21 Nyannor E K D, Williams P, Bedford M R, et al. Corn expressing an Escherichia coli-derived phytase gene: A proof-of-concept nutritional study in pigs. J Anim Sci, 2007, 85: 1946-1952

22 Yu Z Q, Fan B L, Dai Y P, et al. Expression of recombinant human lysozyme in the milk of transgenic mice. Chin Sci Bull, 2003, 48: 2331-2335

23 Valera A, Fillat C, Costa C, et al. Regulated expression of human insulin in the liver of transgenic mice corrects diabetic alterations. FASEB J, 1994, 8: 440-447

24 Ebert K M, Selgrath J P, DiTullio P, et al. Transgenic production of a variant of human tissue-type plasminogen activator in goat milk: Generation of transgenic goats and analysis of expression. Nat Biotechnol, 1991, 9: 835-838

25 Wright G, Carver A, Cottom D, et al. High level expression of active human alpha-1-antitrypsin in the milk of transgenic sheep. Nat Biotechnol, 1991, 9: 830-834

26 Zhang J, Li L, Cai Y, et al. Expression of active recombinant human lactoferrin in the milk of transgenic goats. Protein Expres Purif, 2008, 57: $127-135$

27 Sharma A, Martin M J, Okabe J F, et al. An isologous porcine promoter permits high level expression of human hemoglobin in transgenic swine. Nat Biotechnol, 1994, 12: 55-59 
28 Adiguzel C, Iqbal O, Demir M, et al. European community and US-FDA approval of recombinant human antithrombin produced in genetically altered goats. Clin Appl Thromb-Hem, 2009, 15: 645-651

29 Murakami H, Nagashima H, Takahagi Y, et al. Transgenic pigs expressing human decay-accelerating factor regulated by porcine $\mathrm{MCP}$ gene promoter. Mol Reprod Dev, 2002, 61: 302-311

30 Norman P S, Marsh D G. Human serum albumin and Tween 80 as stabilizers of allergen solutions. J Allergy Clin Immunol, 1978, 62: 314-319

31 Frew A, Chang $\mathrm{J} \mathrm{H}$, Chan $\mathrm{H}$, et al. T-lymphocyte responses to plicatic acid-human serum albumin conjugate in occupational asthma caused by western red cedar. J Allergy Clin Immunol, 1998, 101: $841-847$

32 Xu H L, Zhai H L, Wang F, et al. Cowpea trypsin inhibitor gene (cpti) and its application in insect resistance transgenic plants (in Chinese). J Agri Sci Technol, 2008, 10: 18-27

33 Afshari A, Wetterslev J, Brok J, et al. Antithrombin III in critically ill patients: Systematic review with meta-analysis and trial sequential analysis. BMJ, 2007, 335: 1248-1251

34 Weichel M, Glaser A G, Ballmer-Weber B K, et al. Wheat and maize thioredoxins: A novel cross-reactive cereal allergen family related to baker's asthma. J Allergy Clin Immunol, 2006, 117: 676-681

35 del Val G, Yee B C, Lozano R M, et al. Thioredoxin treatment increases digestibility and lowers allergenicity of milk. J Allergy Clin Immunol, 1999, 103: 690-697

36 Nordlee J A, Taylor S L, Townsend J A, et al. Identification of a Brazilnut allergen in transgenic soybeans. N Engl J Med, 1996, 334: 688-692

37 Centers for Disease Control. Investigation of human health effects associated with potential exposure to genetically modified corn. A report to the US Food and Drug Administration from the Centers for Disease Control and Prevention. http://wwwcdcgov/nceh/ehhe/Cry9Creport/, 2001

38 Ladics G S. Current codex guidelines for assessment of potential protein allergenicity. Food Chem Toxicol, 2008, 46(Suppl 10): S20-S23

39 Dearman R J, Kimber I. Animal models of protein allergenicity: Potential benefits, pitfalls and challenges. Clin Exp Allergy, 2009, 39: $458-468$

40 Herman R A, Song P, Thirumalaiswamyse A. Value of eight-aminoacid matches in predicting the allergenicity status of proteins: An empirical bioinformatic investigation. Clin Mol Allergy, 2009, 7: 9

Open Access This article is distributed under the terms of the Creative Commons Attribution License which permits any use, distribution, and reproduction in any medium, provided the original author(s) and source are credited.

\section{Supporting Information}

Table S1 Summary of plant transforming gene exhibiting no allergenicity and no adverse events

Table S2 Summary of animal transforming gene exhibiting no allergenicity and no adverse events

The supporting information is available online at csb.scichina.com and www.springerlink.com. The supporting materials are published as submitted, without typesetting or editing. The responsibility for scientific accuracy and content remains entirely with the authors. 